\title{
MONOPOLE POTENTIAL AND CONFINING STRINGS IN THE (2+1)-DIMENSIONAL GEORGI-GLASHOW MODEL
}

\author{
DMITRI ANTONOV \\ INFN-Sezione di Pisa, Universitá degli studi di Pisa, Dipartimento di Fisica, Via \\ Buonarroti, 2 - Ed. B - I-56127 Pisa, Italy \\ E-mail: antonov@df.unipi.it
}

\begin{abstract}
Confining strings are investigated in the $(2+1) \mathrm{D}$ Georgi-Glashow model. This is done in the limit when the electric coupling constant is much larger than the square root of the mass of the Higgs field, but much smaller than the vacuum expectation value of this field. The modification of the Debye mass of the dual photon with respect to the case when it is considered to be negligibly small compared to the Higgs mass, is found. Analogous modifications of the potential of monopole densities and string coupling constants are found as well.
\end{abstract}

$(2+1)$ D Georgi-Glashow model is known to be the famous example of a theory allowing for an analytical description of confinement 1 . The confinement mechanism in this model is based on the presence of 't Hooft-Polyakov monopoles 2, whose stochastic fluxes through the contour of the Wilson loop provide the area law. In this talk, we shall discuss string representation of the Wilson loop in this model for the case when, contrary to the compact-QED limit, the Higgs mass is not considered to be infinitely large w.r.t. the Debye mass of the dual photon.

The Euclidean action of the (2+1)D Georgi-Glashow model has the following form

$$
S=\int d^{3} x\left[\frac{1}{4 g^{2}}\left(F_{\mu \nu}^{a}\right)^{2}+\frac{1}{2}\left(D_{\mu} \Phi^{a}\right)^{2}+\frac{\lambda}{4}\left(\left(\Phi^{a}\right)^{2}-\eta^{2}\right)^{2}\right]
$$

where the Higgs field $\Phi^{a}$ transforms by the adjoint representation, and $D_{\mu} \Phi^{a} \equiv \partial_{\mu} \Phi^{a}+\varepsilon^{a b c} A_{\mu}^{b} \Phi^{c}$. In the one-loop approximation, the partition function of this theory reads 3

$$
\mathcal{Z}=1+\sum_{N=1}^{\infty} \frac{\zeta^{N}}{N !}\left[\prod_{i=1}^{N} \int d^{3} z_{i} \sum_{q_{i}= \pm 1}\right] \times
$$

Paris: submitted to World Scientific on November 18, 2018 


$$
\times \exp \left\{-\frac{g_{m}^{2}}{2}\left[\int d^{3} x d^{3} y \rho_{\text {gas }}(\mathbf{x}) D_{0}(\mathbf{x}-\mathbf{y}) \rho_{\mathrm{gas}}(\mathbf{y})-\sum_{\substack{a, b=1 \\ a \neq b}}^{N} D_{m}\left(\mathbf{z}_{a}-\mathbf{z}_{b}\right)\right]\right\} .
$$

Here, $g_{m}$ is the magnetic coupling constant of dimensionality [length $]^{1 / 2}$ related to the electric one $g$ according to the equation $g g_{m}=4 \pi, \rho_{\text {gas }}(\mathbf{x})=$ $\sum_{a=1}^{N} q_{a} \delta\left(\mathbf{x}-\mathbf{z}_{a}\right)$ is the density of monopole gas with $q_{a}$ 's standing for the monopole charges in the units of $g_{m}$. Next, in Eq. (22), $m=\eta \sqrt{2 \lambda}$ is the mass of the Higgs boson and

$$
\zeta=\frac{m_{W}^{7 / 2}}{g} \delta\left(\frac{\lambda}{g^{2}}\right) \mathrm{e}^{-\left(4 \pi / g^{2}\right) m_{W} \epsilon\left(\lambda / g^{2}\right)}
$$

is the statistical weight of a single monopole (else called fugacity) with $m_{W}=$ $g \eta$ being the mass of the $W$-boson. Here, $\epsilon$ is a slowly varying function equal th unity at the origin [i.e. in the Bogomolny-Prasad-Sommerfield (BPS) limit] 6 and $1.787 \ldots$ at infinity $\mathrm{E}$, whereas the function $\delta$ is determined by the loop corrections. Finally, in Eq. (2), $D_{0}(\mathbf{x}) \equiv 1 /(4 \pi|\mathbf{x}|)$ is the Coulomb propagator, and $D_{m}(\mathbf{x}) \equiv \mathrm{e}^{-m|\mathbf{x}|} /(4 \pi|\mathbf{x}|)$ is the propagator of the Higgs boson.

Notice that as it follows from Eq. (2), in the BPS limit, the interaction of two monopoles doubles for opposite and vanishes for equal charges. As far as the opposite limit, $m \rightarrow \infty$, is concerned, we apparently arrive there at the standard compact-QED result 1 .

The effective field theory describing the grand canonical partition function (2) can easily be obtained and reads $\mathrm{B}$

$$
\begin{gathered}
\mathcal{Z}=\int \mathcal{D} \chi \mathcal{D} \psi \times \\
\times \exp \left\{-\int d^{3} x\left[\frac{1}{2}(\nabla \chi)^{2}+\frac{1}{2}(\nabla \psi)^{2}+\frac{m^{2}}{2} \psi^{2}-2 \zeta \mathrm{e}^{g_{m} \psi} \cos \left(g_{m} \chi\right)\right]\right\},
\end{gathered}
$$

where $\chi$ is the dual photon field, whereas the field $\psi$ is an additional one. The latter field can be integrated out in the limit $g \gg \sqrt{m}$, when the exponent in the last term on the R.H.S. of Eq. (1) can be shown 6 to be approximated by the terms not higher than the linear one.

In such a limit, Gaussian integration over the field $\psi$ yields 


$$
\begin{gathered}
\mathcal{Z}=\int \mathcal{D} \chi \exp \left\{-\int d^{3} x\left[\frac{1}{2}(\nabla \chi)^{2}-2 \zeta \cos \left(g_{m} \chi\right)\right]+\right. \\
\left.+2\left(g_{m} \zeta\right)^{2} \int d^{3} x d^{3} y \cos \left(g_{m} \chi(\mathbf{x})\right) D_{m}(\mathbf{x}-\mathbf{y}) \cos \left(g_{m} \chi(\mathbf{y})\right)\right\} .
\end{gathered}
$$

The last term here represents the correction to the standard result 1 . It stems from the fact that the mass of the Higgs field was considered to be not infinitely large compared to the standard Debye mass of the dual photon, $m_{D}=g_{m} \sqrt{2 \zeta}$. The respective correction to $m_{D}$ is positive, and the square of the full mass reads:

$$
M^{2}=m_{D}^{2}\left(1+\frac{m_{D}^{2}}{m^{2}}\right) .
$$

Clearly, this result is valid at $m_{D} \ll m$ and reproduces $m_{D}^{2}$ in the limit $m \rightarrow \infty$.

Another relation between the dimensionful parameters in the model (1)), we shall adapt for our analysis, is $g \ll \eta$. [Clearly, this inequality parallels the requirement that $\eta$ should be large enough to ensure the spontaneous symmetry breaking from $S U(2)$ to $U(1)$.] In particular, from this relation and the inequality $g \gg \sqrt{m}$ we immediately obtain:

$$
\frac{\lambda}{g^{2}} \sim\left(\frac{m}{m_{W}}\right)^{2} \ll\left(\frac{g}{\eta}\right)^{2} \ll 1 .
$$

This means that we are working in the regime of the Georgi-Glashow model close to the BPS limit.

Note further that in the limit $g \gg \sqrt{m}$, the dilute gas approximation holds perfectly. Indeed, this approximation implies that the mean distance between monopoles, equal to $\zeta^{-1 / 3}$, should be much larger than the inverse mass of the $W$-boson. By virtue of Eq. (3) and the fact that the function $\epsilon$ is of the order of unity, we obtain that this requirement is equivalent to the following one:

$$
\sqrt{\frac{\eta}{g}} \delta\left(\frac{\lambda}{g^{2}}\right) \mathrm{e}^{-4 \pi \eta / g} \ll 1 .
$$

Although at $\lambda \ll g^{2}[c f$. Eq. (7)], the function $\delta$ grows, the speed of this growth is so that at $g \ll \eta$, the L.H.S. of Eq. (8) remains exponentially small 
Another consequence of this fact is that in the regime of the Georgi-Glashow model under discussion, the Debye mass of the dual photon, $m_{D}$, remains exponentially small as well. In particular, the inequality $m_{D} \ll m$, under which the full mass (6) was derived, holds due to this smallness. Also, due to the same reason, the mean field approximation, under which the effective field theory (1) is applicable, remains valid as well with the exponential accuracy. Indeed, this approximation implies that in the Debye volume ${ }^{a} m_{D}^{-3}$ there should contain many particles 1 . Since the average density of monopoles is equal to $2 \zeta$ [which can be seen either by calculating it directly according to the formula $V^{-1} \partial \ln \mathcal{Z} / \partial \ln \zeta$, applied to Eq. (5) at $m_{D} \ll m$, or from the remark following after Eq. (11) below], we arrive at the requirement $\zeta m_{D}^{-3} \gg 1$. Substituting the above-obtained value for $m_{D}$, we see that the criterion of applicability of the mean field approximation reads $g^{3} \gg \sqrt{\zeta}$. Owing to the above-discussed exponential smallness of $\zeta$, this inequality is satisfied.

One can now derive the potential of (dynamical) monopole densities corresponding to the partition function (5). Referring the reader for the details to the original paper $\mathrm{G}$, we present here the final expression for the partition function (5) in terms of the integral over these densities:

$$
\mathcal{Z}=\int \mathcal{D} \rho \exp \left\{-\left[\frac{g_{m}^{2}}{2} \int d^{3} x d^{3} y \rho(\mathbf{x}) D_{0}(\mathbf{x}-\mathbf{y}) \rho(\mathbf{y})+V[\rho]\right]\right\} .
$$

The monopole potential $V[\rho]$ here reads

$$
\begin{gathered}
V[\rho]=\int d^{3} x\left[\rho \operatorname{arcsinh} \varrho-2 \zeta \sqrt{1+\varrho^{2}}\right]- \\
-2\left(g_{m} \zeta\right)^{2} \int d^{3} x d^{3} y \sqrt{1+\varrho^{2}(\mathbf{x})} D_{m}(\mathbf{x}-\mathbf{y}) \sqrt{1+\varrho^{2}(\mathbf{y})},
\end{gathered}
$$

where $\varrho \equiv \rho /(2 \zeta)$. The last term on the R.H.S. of this equation is again a leading $\left(m_{D} / m\right)$-correction to the respective $(m=\infty)$-expression. In the dilute gas approximation, $|\rho| \ll \zeta$, Eq. (10) becomes a simple quadratic functional:

$$
V[\rho] \rightarrow \frac{1}{2}\left[\frac{1}{2 \zeta}-\left(\frac{g_{m}}{m}\right)^{2}\right] \int d^{3} x \rho^{2} \simeq \frac{g_{m}^{2}}{2 M^{2}} \int d^{3} x \rho^{2},
$$

where the last equality is implied within the leading $\left(m_{D} / m\right)$-approximation adapted. This leads to the simple expression for the generating functional of correlators of the monopole densities within these two approximations:

${ }^{a}$ In this discussion, the difference between $m_{D}$ and $M$ is unimportant. 


$$
\begin{gathered}
\mathcal{Z}[j] \equiv \int \mathcal{D} \rho \times \\
\times \exp \left\{-\left[\frac{g_{m}^{2}}{2} \int d^{3} x d^{3} y \rho(\mathbf{x}) D_{0}(\mathbf{x}-\mathbf{y}) \rho(\mathbf{y})+\frac{g_{m}^{2}}{2 M^{2}} \int d^{3} x \rho^{2}+\int d^{3} x j \rho\right]\right\}= \\
=\exp \left[-\frac{M^{2}}{2 g_{m}^{2}} \int d^{3} x d^{3} y j(\mathbf{x}) j(\mathbf{y}) \partial^{2} D_{M}(\mathbf{x}-\mathbf{y})\right]
\end{gathered}
$$

[Sending for a while $m_{D}$ to zero (since it is exponentially small), we get from Eq. (11): $\langle\rho(\mathbf{x}) \rho(0)\rangle=2 \zeta \delta(\mathbf{x})$. This means that with the exponential accuracy the average density of monopoles is equal to $2 \zeta$, which can also be seen directly from the $(|\rho| \ll \zeta)$-limit of Eq. (10).] In particular, the Wilson loop reads:

$$
\begin{gathered}
\langle W(\mathcal{C})\rangle=\langle W(\mathcal{C})\rangle_{\text {free }} \mathcal{Z}[i \eta]= \\
=\exp \left\{-\frac{g^{2}}{2}\left[\frac{M^{2}}{2} \int_{\Sigma} d \sigma_{\mu \nu}(\mathbf{x}) \int_{\Sigma} d \sigma_{\mu \nu}(\mathbf{y})+\oint_{\mathcal{C}} d x_{\mu} \oint_{\mathcal{C}} d y_{\mu}\right] D_{M}(\mathbf{x}-\mathbf{y})\right\} .
\end{gathered}
$$

This equation can straightforwardly be derived by making use of the formula

$$
\partial_{\mu} \eta(\mathbf{x})=2 \pi \varepsilon_{\mu \nu \lambda}\left[2 \partial_{\nu}^{x} \oint_{\mathcal{C}} d y_{\lambda} D_{0}(\mathbf{x}-\mathbf{y})-\int_{\Sigma} d \sigma_{\nu \lambda}(\mathbf{y}) \delta(\mathbf{x}-\mathbf{y})\right] .
$$

Here, $\eta(\mathbf{x})=2 \pi \varepsilon_{\mu \nu \lambda} \partial_{\mu}^{x} \int_{\Sigma} d \sigma_{\nu \lambda}(\mathbf{y}) D_{0}(\mathbf{x}-\mathbf{y})$ is the solid angle under which an arbitrary surface $\Sigma$ spanned by the contour $\mathcal{C}$ shows up to the observer located at the point $\mathbf{x}$. Also, in Eq. (12), $\langle W(\mathcal{C})\rangle_{\text {free }}=$ $\exp \left[-\frac{g^{2}}{2} \oint_{\mathcal{C}} d x_{\mu} \oint_{\mathcal{C}} d y_{\mu} D_{0}(\mathbf{x}-\mathbf{y})\right]$ is the contribution of the free photons to the Wilson loop. The explicit $\Sigma$-dependence of the R.H.S. of Eq. (12) appearing in the dilute gas approximation becomes eliminated by the summation over branches of the arcsinh-function in the full monopole action (9)-(10). This is the main principle of correspondence between fields strings, proposed 
for compact QED in Ref. $\mathrm{O}$ in the language of the Kalb-Ramond field $h_{\mu \nu}$, $\varepsilon_{\mu \nu \lambda} \partial_{\mu} h_{\nu \lambda} \propto \rho$.

As far as the string tension and the inverse coupling constant of the rigidity term $\mathrm{O}$ are concerned, those can be evaluated upon the derivative expansion of the $\sum$-dependent part of Eq. (12). By virtue of the general formulae from Ref. 10 we obtain

$$
\sigma=4 \pi g^{2} M \text { and } \alpha^{-1}=-\frac{\pi g^{2}}{2 M}
$$

respectively. Clearly, both of these quantities represent the modifications of the standard ones, corresponding to the limit when $m$ is considered to be infinitely large w.r.t. $m_{D}$. The standard expressions follow from Eq. (13) upon the substitution into this equation $M \rightarrow m_{D}$.

\section{Acknowledgments}

The author is grateful to Prof. A. Di Giacomo for useful discussions and to INFN for the financial support. He was also partially supported by the INTAS grant Open Call 2000, project No. 110. And last but not least, the author acknowledges the organizers of the Sixth Workshop on Nonperturbative QCD (American University of Paris, Paris, France, June 2001) for an opportunity to present these results in a very stimulating atmosphere.

\section{References}

1. A.M. Polyakov, Nucl. Phys. B 120, 429 (1977).

2. G. 't Hooft, Nucl. Phys. B 79, 276 (1974); A.M. Polyakov, JETP Lett. 20, 194 (1974).

3. K. Dietz and Th. Filk, Nucl. Phys. B 164, 536 (1980).

4. M.K. Prasad and C.M. Sommerfield, Phys. Rev. Lett. 35, 760 (1975); E.B. Bogomolny, Sov. J. Nucl. Phys. 24, 449 (1976).

5. T.W. Kirkman and C.K. Zachos, Phys. Rev. D 24, 999 (1981).

6. N. Agasian and D. Antonov, JHEP 06, 058 (2001).

7. V.G. Kiselev and K.G. Selivanov, Phys. Lett. B 213, 165 (1988).

8. A.M. Polyakov, Nucl. Phys. B 486, 23 (1997).

9. A.M. Polyakov, Nucl. Phys. B 268, 406 (1986); H. Kleinert, Phys. Lett. B 174, 335 (1986).

10. D.V. Antonov, D. Ebert, and Yu.A. Simonov, Mod. Phys. Lett. A 11, 1905 (1996). 